\title{
Prevalencia de Ninfas de Linguatula serrata en Ganado Bovino en Camales de Lima, Perú
}

\author{
Prevalence of Linguatula Serrata Nymphs in Cattle in Abattoirs in Lima, Peru \\ Julia Castro ${ }^{1}$, Asucena Naupay ${ }^{2}$, Narda Fajardo ${ }^{1}$, Giannina Trevejo ${ }^{1}$, Víctor Almeyda ${ }^{1}$, \\ Esperanza Fajardo ${ }^{1}$, Karina Faustino ${ }^{1}$
}

\section{Resumen}

La linguatulosis es una zoonosis causada por Linguatula serrata, parásito que puede afectar al hombre en su estadio ninfal y adulto, siendo los principales mecanismos de transmisión el contacto con el perro doméstico y la ingesta de vísceras infectadas mal cocidas de bovinos. El presente estudio tuvo como objetivo determinar la prevalencia, abundancia e intensidad media del estadio ninfal de L. serrata en ganglios linfáticos mesentéricos yeyuno-iliacos de reses beneficiadas en dos camales de la ciudad de Lima, Perú, procedentes de los departamentos de Puno, Junín, La Libertad, Amazonas, Pasco y Lima. Se recolectaron 3576 ganglios de 368 animales, se les retiró la grasa circundante y refrigeraron a $5{ }^{\circ} \mathrm{C}$ hasta su procesamiento. Se realizaron cortes longitudinales en ganglios con microabscesos, granulomas calcificados o con nódulos de color amarillo, verde o gris. Las ninfas colectadas fueron fijadas y montadas. Resultados de la morfometría de las ninfas indicaron promedios de $4.95 \mathrm{~mm}$ de largo total, $1.39 \mathrm{~mm}$ de ancho anterior y 0.76 $\mathrm{mm}$ de ancho posterior. La prevalencia de infección por ninfas de L. serrata fue de $5.97 \%$, siendo más frecuente en animales de 4-5 años de edad. El ganado más afectado procedía de Chachapoyas, Amazonas (13/121) y de Huancayo, Junín (6/70). La abundancia media fue de $0.14(0-2)$ y la intensidad media de $2.5(2-3)$.

Palabras clave: Linguatula serrata, ganglios linfáticos mesentéricos, prevalencia, ganado bovino, camal

\section{Abstract}

Linguatulosis is a zoonosis caused by Linguatula serrata. The nymph and adult stages of the parasite can affect humans, and the man main mechanisms of transmission is through contact with domestic dogs and ingestion of uncooked infected cattle viscera. This study aimed to determine the prevalence, mean abundance, and the mean intensity

\footnotetext{
${ }^{1}$ Laboratorio de Artrópodos Parásitos y Vectores, ${ }^{2}$ Laboratorio de Parasitología Humana y Animal, Facultad de Ciencias Biológicas, Universidad Nacional Mayor de San Marcos, Lima, Perú

${ }^{3}$ E-mail:jch4920@hotmail.com
}

Recibido: 18 de noviembre de 2014

Aceptado para publicación: 23 de febrero de 2015 
of the nymphal stage of $L$. serrata in mesenteric lymph nodes of cattle slaughtered in two abattoirs in Lima, Peru. The animals were brought from the departments of Puno, Junín, La Libertad, Amazonas, Pasco and Lima. A total of 3576 lymph nodes from 368 cattle were collected, fat was removed and stored at $5{ }^{\circ} \mathrm{C}$. Nodes with micro abscesses, calcified granulomas and yellow, green or gray nodules were selected and longitudinal cuts were done. Nymphs were fixed and mounted for evaluation. Results of the morphometry showed averages of $4.95 \mathrm{~mm}$ total length, $1.39 \mathrm{~mm}$ anterior width and $0.76 \mathrm{~mm}$ posterior width. The prevalence of infection by nymphs of $L$. serrata was $5.97 \%$, especially in 4-5 years old animals. The most frequent affected cattle were from Chachapoyas, Amazonas (13/121) and Huancayo, Junín (6/70). The mean abundance was $0.14(0-2)$ and the mean intensity was $2.5(2-3)$.

Key words: Linguatula serrata, mesenteric lymph nodes, prevalence, cattle, slaughterhouse

\section{INTRODUCCIÓN}

La linguatulosis o pentastomiosis es una zoonosis parasitaria causada por dos especies parasitarias: Linguatula serrata y Armillifer armillatus (Acha y Szyfres, 2003). Son endoparásitos patógenos, hematófagos, diheteroxenos y eurígenos, que comparten similitudes con los artrópodos y anélidos, pero actualmente se encuentran dentro del Phylum Arthropoda debido a estudios ultraestructurales, embriológicos y genéticos (Meyers et al., 1976). Presentan cuatro estadios evolutivos: huevo, larva, ninfa y adulto. Las formas ninfales han sido localizadas en el hígado, pulmones, riñones, corazón y ganglios de rumiantes, incluyendo al bovino, que actúan como hospederos intermediarios en el ciclo evolutivo (Berger y Marr, 2006). Los hospederos definitivos son los cánidos, pero no tienen especificidad de hospedero, pudiendo el humano comportarse como hospedero intermediario o definitivo accidental.

El ciclo de L. serrata fue descrito por Hobmaier y Hobmaier (1940). El adulto vive en los conductos respiratorios del perro, zorro, lobos, caballos, cabras y ovejas (hospederos definitivos - HD) donde depositan huevos, que son expulsados con las secreciones mucosas, contaminando la vegetación y el agua. Los huevos, al ser deglutidos por un herbívoro (hospedero intermediario - HI), eclosionan liberando una larva acariforme que penetra a través de la pared intestinal y se aloja en las vísceras, especialmente en nódulos mesentéricos, hígado y pulmones, donde se cubre por una cubierta elaborada por el hospedero intermediario. La larva pasa por 6-9 mudas para dar lugar a la ninfa infectiva que se desplaza hasta la cavidad abdominal o pleural del HI donde se enquista. Cuando el HI o sus vísceras son ingeridos por el HD, la ninfa abandona el quiste y se fija en la membrana mucosa de la boca, donde migran hacia las cavidades nasales y se transforman en adultos. Las hembras después de la cópula comienzan a depositar huevos durante seis meses. Los parásitos adultos pueden vivir hasta dos años, después de los cuales se produce, por lo general, la recuperación del HD (Soulsby, 1987).

La infección humana es poco frecuente y la mayoría de casos han ocurrido en regiones tropicales y subtropicales. La infección se produce por el estadio ninfal de $L$. serrata cuando se ingiere hígado $u$ otras vísceras crudas o mal cocidas de herbívoros parasitados (Baird et al., 1988; Tappe et al., 2006; Mohammadi et al., 2008; Hamid et al., 2012), pero también puede desarrollar la forma visceral al ingerir accidentalmente verduras o aguas contaminadas con heces de cánidos, comportándose como hospedero 
intermediario (Sielfeld, 2000). Los casos humanos de infestación intraocular son pocos, y algunos han sido reportados en EEUU e Israel (Lang et al., 1987) y Ecuador (Lazo et al., 1999).

El ganado bovino alberga los estadios ninfales del parásito, causando durante su migración lesiones como granulomas y nódulos en los ganglios mesentéricos, hígado, riñones, corazón y pulmones. Las lesiones son debidas a las laceraciones causadas por los ganchos de las fases larvarias y por la microbiota intestinal que arrastran hacia los órganos. La presencia de nódulos en intestinos los dejan inutilizables para la industria de embutidos. Asimismo, infestaciones masivas de linguatulosis ninfal pueden ocasionar neumonías o hepatitis, algunas de curso mortal (Guerrero, 2011).

En la literatura existente, los estudios se refieren a decomisos de hígados de bovinos infectados por ser el órgano de mayor comercialización y porque las infecciones masivas con estadios ninfales de $L$. serrata provocan la muerte de bovinos jóvenes. Así, en Chile, Poo (1971) reporta $0.8 \%$ de hígados afectados dentro de 483 hígados decomisados por diversas razones; Garcinuño y Gonzales (1977) en un examen de 1000 hígados en el camal de Valdivia encontraron 59 casos $(5.9 \%)$ de linguatulosis; y Valenzuela et al. (1995) en 1011 hígados decomisados hallaron $16.6 \%$ contaminados con L. serrata.

La linguatulosis es poco conocida en el Perú. El único estudio realizado en Lima ha sido el de Dodero (1956), quien examinó 120 perros callejeros en las inmediaciones de los camales, reportando por primera vez el hallazgo del estadio adulto de $L$. serrata en las fosas nasales de 7/30 perros del distrito de Surco, 1/10 del distrito de Barranco y 5/20 del Centro de Instrucción Militar del Perú (CIM). También inspeccionó 500 bovinos del camal del CIM, donde $18 \%$ presentaban el estadio larvario, principalmente en los ganglios mesentéricos.
No se dispone de reportes actualizados sobre esta parasitosis, ni sobre el riesgo epidemiológico para el hombre. El presente estudio tuvo como objetivo determinar la prevalencia de ninfas de $L$. serrata en ganglios mesentéricos de ganado bovino beneficiado en dos camales de la ciudad de Lima, con la finalidad de aportar nuevos antecedentes acerca del parasitismo por $L$. serrata en ganado bovino que se beneficia para consumo humano.

\section{Materiales y Métodos}

Se recolectaron entre mayo de 2010 y junio de 2011, cadenas de ganglios mesentéricos cubiertos con grasa abdominal de 368 bovinos beneficiados en los camales frigoríficos «La Colonial» $\mathrm{y}$ «Garagay», de la ciudad de Lima. Los animales procedían de los departamentos de Puno, Junín, La Libertad, Amazonas, Pasco y Lima, Perú. Se trabajó con ganglios mesentéricos por ser una de las localizaciones frecuentes de nifas de L. serrata y por su bajo costo de adquisición.

Se retiró la grasa de los ganglios (Fig. 1a) y se transportaron al Laboratorio de Control de Artrópodos y Vectores de la Facultad de Ciencias Biológicas, Universidad Nacional Mayor de San Marcos, Lima, donde se mantuvieron a $5{ }^{\circ} \mathrm{C}$.

Se analizaron 3576 ganglios mesentéricos. Los focos infecciosos localizados en la superficie de los ganglios se examinaron macroscópicamente, seleccionándose aquellos que evidenciaron micro-abscesos, granulomas calcificados o presencia de nódulos de color pardo-amarillento, verde o gris (Fig. 1b).

Se realizaron cortes longitudinales en los ganglios seleccionados para ubicar posibles formas ninfales que se observan como puntos cristalinos a través de la cápsula. Las ninfas se aislaron con pinceles y se colocaron en viales con solución salina para la observa- 

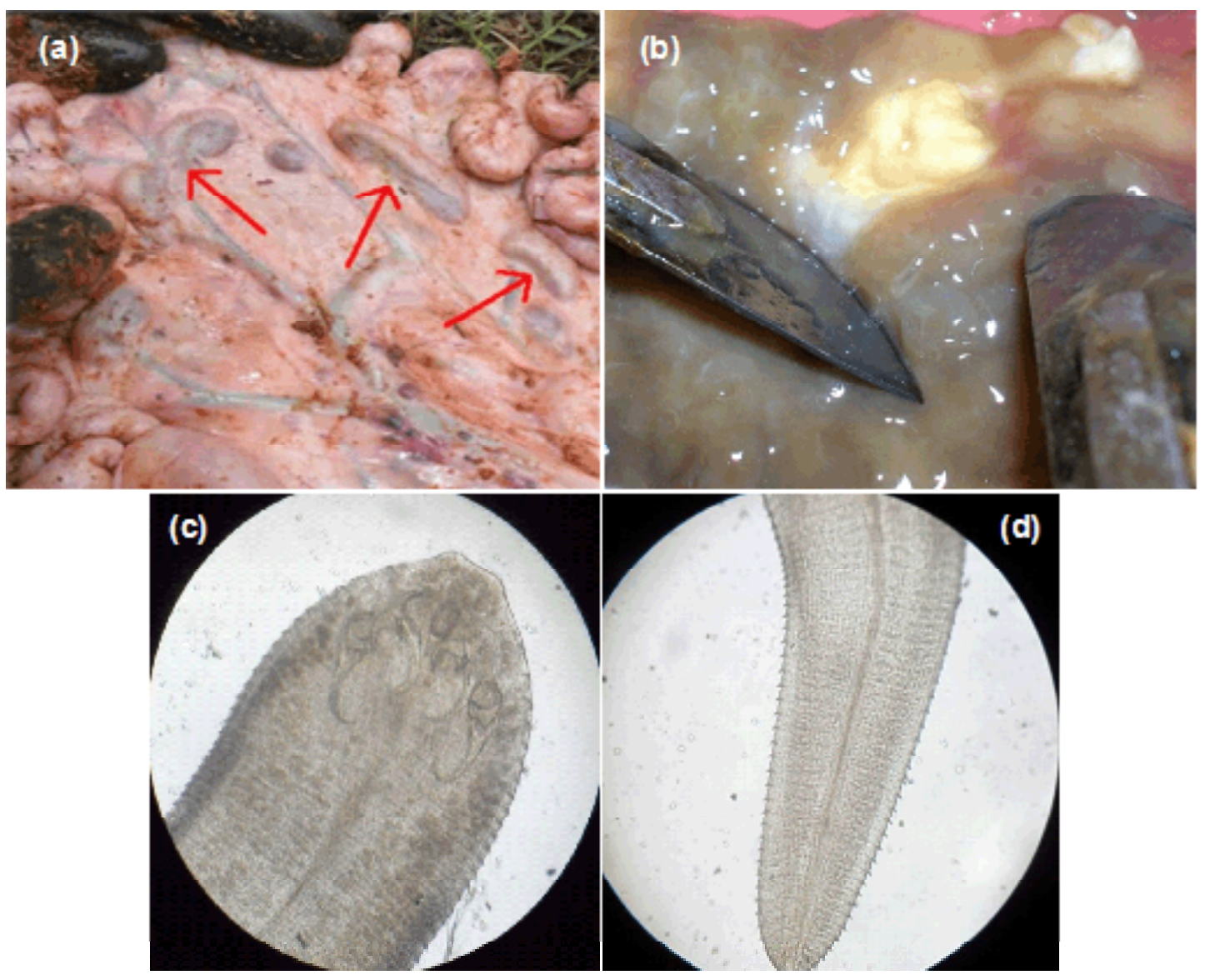

Figura 1. (a) Ganglios mesentéricos de bovino; (b) Nódulos de color pardo-amarillento en ganglios mesentéricos; Extremidad anterior (c) y posterior (d) de la ninfa de Linguatula serrata $4 \mathrm{x}$

ción microscópica de sus características morfológicas y morfometría. El diagnóstico específico se realizó a través de las características morfométricas de las ninfas como el largo total, ancho anterior y posterior, número de segmentos abdominales y el patrón de disposición de las espinas y ganchos, siguiendo la metodología de Tajik y Sabet Jalali (2010) (Fig. 1c,d). Los ganglios infectados se fijaron en formol al $10 \%$ para su posterior estudio histológico.

La fijación de las ninfas se realizó por el método de prensado y se conservaron en formol 10\% hasta la coloración del material.
Para obtener un montaje adecuado de las ninfas, se procedió según lo establecido por Lazo et al. (1999).

Para hallar la prevalencia se aplicó la prueba de hipótesis para proporciones bajo distribución normal, para un nivel de confianza $(1-\alpha)$ del $97.5 \%$ y valor de normalidad o punto crítico $Z_{\alpha}=Z_{0.035}=-1.96$. Se determinó la abundancia media (total de parásitos/ población total) y la intensidad media (total de parásitos/población parasitada) del parasitismo ocasionado por ninfas de $L$. serrata, siguiendo la terminología ecológica de Bush et al. (1997). 
Cuadro 1. Casos positivos con ninfas de Linguatula serrata en ganado bovino beneficiado en camales de Lima, Perú (2010-2011)

\begin{tabular}{llccc}
\hline \multirow{2}{*}{ Camal } & Procedencia & \multicolumn{2}{c}{ Ganado } & \multirow{2}{*}{$\begin{array}{c}\text { Edad } \\
\text { (años) }\end{array}$} \\
\cline { 3 - 4 } La Colonial & Puno & 34 & 1 & 3 a 4 \\
& Chachapoyas & 121 & 13 & 4 a 5 \\
& Pozuzo & 63 & 1 & 3 \\
& Trujillo & 15 & 0 & 4 \\
& Huancayo & 70 & 6 & 4 a 5 \\
\cline { 2 - 4 } & Sub-total & 303 & 21 & \\
\hline \multirow{6}{*}{ Garagay } & Huancayo & 45 & 1 & 4 \\
& Lima & 20 & 0 & 3 a 4 \\
\cline { 2 - 4 } & Sub-total & 61 & 1 & \\
\hline
\end{tabular}

\section{Resultados}

Se recolectaron 303 muestras en el camal «La Colonial» y 65 muestras en el camal «Garagay», donde la mayoría de los animales tenía entre 3 y 5 años de edad (Cuadro 1). En el caso de los animales beneficiados en «La Colonial», las mayores frecuencias de ganglios con ninfas de L. serrata se observaron en bovinos procedentes de Chachapoyas, Amazonas (13/121) y de Huancayo, Junín (6/70). Por otro lado, hubo muy pocos casos positivos en los animales de otras procedencias o en aquellos beneficiados en el camal «Garagay».

El porcentaje de prevalencia de ninfas de $L$. serrata fue de $5.97 \%$, especialmente en animales de 4 a 5 años de edad. La abundancia media fue de $0.14(0-2)$ y la intensidad media de 2.5 (2-3). La morfometría promedio de las ninfas fue de $4.95 \mathrm{~mm}$ de largo total (L), $1.39 \mathrm{~mm}$ de ancho anterior (AA) y $0.76 \mathrm{~mm}$ de ancho posterior (AP).

\section{Discusión}

Las prevalencias de larvas de L. serrata reportadas son bastante disímiles. Oryan et al. (2008) hallaron una prevalencia de 52.5\% en nódulos mesentéricos de 200 ovejas y Saeid et al. (2010) obtuvieron una prevalencia de $16.2 \%$ en nódulos mediastinales y mesentéricos de 450 bovinos, ambos en Irán, mientras que en Chile, Poo (1971) reportó $0.8 \%$, Garcinuño y Gonzales (1977) hallaron $5.9 \%$ y Valenzuela et al. (1995) encontraron $16.6 \%$ de prevalencia de $L$. serrata, donde estos investigadores trabajaron con hígados de bovinos.

Los resultados del presente estudio indican una baja prevalencia de $L$. serrata (5.97\%), donde la mayor frecuencia de casos se presentó en bovinos procedentes de Chachapoyas (13/121) y Pozuzo (6/70), coincidiendo en la edad de los animales (4 a 5 años), lo cual podría establecer una relación parásito-edad. La relativa baja prevalencia 
encontrada podría deberse a que los estadios ninfales en ganglios linfáticos no es la localización de mayor frecuencia, sino en el hígado (Garcinuño y Gonzales, 1977; Valenzuela et al., 1995). De acuerdo al ciclo biológico, el parásito podría alojarse en otros órganos que no fueron motivo del presente estudio.

En estudios de morfometría de la ninfa de $L$. serrata en la superficie ocular de un humano, las medidas obtenidas por Koehsler et al. (2011) fueron de $\mathrm{L}=4.5 \mathrm{~mm}, \mathrm{AA}=$ $0.9 \mathrm{~mm}$ y $\mathrm{AP}=0.35 \mathrm{~mm}$; siendo más pequeñas que las obtenidas en el presente estudio $(\mathrm{L}=4.95 \mathrm{~mm} . \mathrm{AA}=1.39 \mathrm{~mm}$ y $\mathrm{AP}=0.76 \mathrm{~mm})$. Esto podría ser un indicio de la especificidad de hospedero y de su localización.

Se encontraron entre 1 a 4 ninfas por ganglio, mientras que Dodero (1956) encontró entre 1 a 6 ninfas; no obstante, es importante considerar el número de animales examinados y la metodología de disección de los ganglios para poder hacer una mejor comparación.

Es posible que en las zonas ganaderas de donde provenían los animales del estudio no se tomen las medidas adecuadas de control contra el parasitismo, y que los perros, hospederos definitivos de este parásito, mantengan la infección y contaminen el ganado. Además, los antiparasitarios contra parásitos de perros no describen acción alguna contra L. serrata. Por lo tanto, se sugiere que la población reciba educación sanitaria sobre las desventajas de comer carne cruda o hígado insuficientemente cocido $\mathrm{u}$ otros órganos internos de los herbívoros. L. serrata representa un riesgo para la salud humana y animal, recomendándose tomar en cuenta esta infección en los lugares donde se realiza el beneficio del ganado.

\section{Conclusiones}

- El ganado bovino beneficiado para consumo humano en los camales de Lima presenta infección por ninfas de
Linguatula serrata, representando un potencial factor de riesgo, pues su presencia en ganglios linfáticos sugiere que podrían encontrarse en otras localizaciones (hígado, pulmones).

- El ganado más afectado procedía de la zona de Chachapoyas, Amazonas y de Huancayo, Junín, teniendo estos animales entre 4 a 5 años de edad.

\section{Literatura Citada}

1. Acha P, Szyfres B. 2003. Parasitosis. Sección C: Artrópodos. En: Zoonosis y enfermedades transmisibles comunes al hombre y a los animales. $3^{\mathrm{a}}$ ed. Washington DC: OPS. p 369-374.

2. Baird J, Kassebaum L, Ludwig G. 1988. Hepatic granuloma in a man from North America caused by a nymph of Linguatula serrata. Pathology 20: 198199.

3. Berger S, Marr J. 2006. Human parasitic diseases. Sourcebook. USA: Jones \& Bartlett Publishers. 294 p.

4. Bush A, Lafferty K, Lotz J, Shostak A. 1997. Parasitology meets ecology on its own terms: Margolic et al. revisited. J Parasitology 83: 575-583.

5. Dodero O. 1956. Contribución al estudio de la incidencia de la Linguatula serrata en el Canis familiaris y en el Bos taurus. Tesis Bachiller Medicina Veterinaria. Lima: Univ Nacional Mayor de San Marcos. Lima. 59 p.

6. Garcinuño L, Gonzales H. 1977. Linguatulosis hepática en bovinos de Valdivia. Arch Med Vet 9: 62-65.

7. Guerrero C. 2011. Epidemiología y control de la linguatulosis bovina. En: Quiroz H, Figueroa J, Ibarra F, López M (eds). Epidemiología de las enfermedades parasitarias en animales domésticos. p 637642.

8. Hamid T, Hossein Y, Mehran B, Masood F, Hamid E. 2012. A case report of Linguatula serrata infestation from rural area of Isfahan city, Iran. Adv 
Biomed Res 1: 42. doi: 10.4103/22779175.100142

9. Hobmaier A, Hobmaier M. 1940. On the life-cycle of Linguatula rhinaria. Am J Trop Med 20: 199-210.

10. Koehsler M, Walochnik J, Georgopoulos $M$, Pruente $C$, Boeckeler W, Auer H, BarisaniAsenbauer T. 2011. Linguatula serrata tongue worm in human eye, Austria. Emerg Infec Dis 17: 870-872. doi: 10.3201/eid1705.100790

11. Lang Y, Garzozi H, Epstein Z, Barkay $S$, Gold D, Lengy J. 1987. Intraocular pentastomiasis causing unilateral glaucoma. Br J Ophthalmol 71: 391-395. doi: 10.1136/bjo.71.5.391

12. Lazo R, Hidalgo E, Lazo E, Bermeo A, Llaguno M, Murillo J, Teixeira $V$. 1999. Ocular linguatiliasis in Ecuador: case report and morphometric study of the larva of Linguatula serrata. Am J Trop Med Hyg 60: 405-409

13. Mohammadi G, Mobedi I, Ariaiepour M, Pourmohammadi Z, Bidaki MD. 2008. A case report of nasopharyngeal linguatuliasis in Tehran, Iran and characterization of the isolated Linguatula serrata. Iranian J Parasitol 3(1): 53-55.

14. Meyers W, Naefie R, Connor D. 1976. Pentostomiasis. In: Binford H, Connor D (eds). Pathology of tropical and extraordinary diseases. Washington DC, USA: Armed Forces Institute of Pathology. p 546-550.
15. Oryan A, Sadjjadi S, Mehrabani D, Rezaei M. 2008. The status of Linguatula serrata infection of stray dogs in Shiraz, Iran. Comp Clin Pathol 17: 55-60. doi: 10.1007/s00580-007-0707-x

16. Poo L. 1971. Estudio anatomopatológico de los hígados de bovino en el matadero Socoagro de Valdivia. Tesis de Médico Veterinario. Valdivia, Chile: Univ Austral de Chile. 30 p.

17. Saeid R, Nourollahi F, Kheirandish $R$, Norouzi E, Saeid F. 2010. The prevalence of Linguatula serrata nymphs in mesenteric lymph nodes in cattle. Am J Anim Vet Sci 5(2): 155-158.

18. Soulsby E. 1987. Parasitología y enfermedades parasitarias en los animales domésticos. $7^{\mathrm{a}}$ ed. México: Interamericana. $823 \mathrm{p}$.

19. Tajik H, Sabet Jalali. 2010. Linguatula serrata prevalence and morphometrical features: abattoir survey on water buffaloes in Iran. Ital J Anim Sci 9(e65): 348-351. doi: http://dx.doi.org/ 10.4081/ijas.2010.e65

20. Tappe D, Winzer R, Büttner D, Ströbel P, Stich A, Klinker H, Frosch M. 2006. Linguatuliasis in Germany. Emerg Infect Dis 12: 1034-1036. doi: 10.3201/ eid1206.051413

21. Valenzuela G, Bascuñan M, Bayer L, Ernst S. 1995. Infecciones por Linguatula serrata (Frohlich 1789) en hígados de bovinos. Arch Med Vet 27: 29-34. 